Trinity University

Digital Commons @ Trinity

Mathematics Faculty Research

Mathematics Department

$8-2004$

\title{
Basin of Attraction of Periodic Orbits of Maps on the Real Line
}

Saber Elaydi

TrinityUniversity, selaydi@trinity.edu

Robert J. Sacker

Follow this and additional works at: https://digitalcommons.trinity.edu/math_faculty

Part of the Mathematics Commons

\section{Repository Citation}

Elaydi, S., \& Sacker, R. (2004). Basin of attraction of periodic orbits of maps on the real line. Journal of Difference Equations and Applications, 10(10), 881-888. doi:10.1080/10236190410001731443

This Post-Print is brought to you for free and open access by the Mathematics Department at Digital Commons @ Trinity. It has been accepted for inclusion in Mathematics Faculty Research by an authorized administrator of Digital Commons @ Trinity. For more information, please contact jcostanz@trinity.edu. 


\title{
The basin of attraction of one-dimensional maps
}

\author{
S. Elaydi and R. Sacker
}

\section{Introduction}

This paper is motivated by a conjecture due to the first author and A. Yakubu [6] concerning the Ricker's model [10] [4]. It was conjectured that the basin of attraction of an attracting $2^{k}$-cycle is $(0, \infty) \backslash E$, where $E$ is the set of all eventually $2^{r}$-periodic points, $0 \leq r<k[6]$. In this paper we prove this conjecture and much more. In fact we prove the same result for continuous maps on compact intervals. The results are then easily extended to bounded continuous maps on closed intervals. Our results cover the Ricker's map $R(x)=x e^{p-x}$ on $[0, \infty)[10]$ as well as the ever popular logistic map $F_{\mu}(x)=$ $\mu x(1-x)$. The importance of our results stems from the fact that while fixed points of continuous maps can be globally attracting, it is not the case for periodic points. Elaydi and Yakubu [7] have shown that there are no globally attracting $r$-cycles, $r>1$, in a connected metric spaces. For example, in the Ricker's model the fixed point $x^{*}=p$ is globally attracting for $0<p<p_{1}$, where $p_{1}=2$. However, for $2<p<p_{2}$, where $p_{2} \approx 2.5264$, the attracting 2-cycle is not globally attracting and in fact it is shown here that the basin of attraction of the attracting 2-cycle is the complement of the set of all eventually fixed points. This period-doubling bifurcation occurs at the infinite sequence of parameters $p_{n}$, with $\lim _{n \rightarrow \infty} p_{n}=p_{\infty}$ where $p_{\infty} \approx 2.6294$. A similar analysis holds true for the logistic map. In section 3 we extend this analysis to a class of maps we call quasi-unimodal maps.

The Ricker's map $R(x)$ and the logistic map $F_{\mu}(x)$ are examples of onedimensional maps $f$ that give rise to a difference equation

$$
x_{n+1}=f\left(x_{n}\right)
$$


where $x_{n}$ may represent a population size in generation $n$. Equation (1.1) models a simple population with seasonal breeding whose generations do not overlap such as orchard pests, temperate zone insects, invertebrate populations, a variety of fish and much more [9], [10].

We now introduce some definitions and preliminaries.

Standing Notation: In this paper $\mathbb{Z}^{+}$denotes the set of non negative integers and $f^{n}=f \circ f \circ \ldots \circ f$ is the nth composition of $\mathrm{f}$.

Definition 1.1. Let $x^{*}$ be a fixed point of a map $f$. Then $z$ is said to be an eventually fixed point relative to $x^{*}$ if $f^{r}(z)=x^{*}$, for some $r \in \mathbb{Z}^{+}$. The set of all eventually fixed points of $x^{*}$ is denoted by $E F_{x^{*}}$.

Eventually $k$-periodic points are defined similarly. If $\bar{x}$ is a $k$-periodic point, then $z$ is said to be an eventually $k$-periodic point relative to $\bar{x}$ if $f^{s}(z)=\bar{x}$, for some $s \in \mathbb{Z}^{+}$. The set of all eventually $k$-periodic points of an $k$-periodic point $\bar{x}$ is denoted by $E P_{\bar{x}}$. Notice that for a $k$-cycle $\left\{\bar{x}_{1}, \bar{x}_{2}, \ldots, \bar{x}_{k}\right\}$

$$
E P_{\bar{x}_{1}}=E P_{\bar{x}_{2}}=\ldots=E P_{\bar{x}_{k}} .
$$

Definition 1.2. Let $x^{*}$ be a fixed point of a map $f$. Then the basin of attraction (or the stable set) $W^{s}\left(x^{*}\right)$ of $x^{*}$ is defined as

$$
W^{s}\left(x^{*}\right)=\left\{x: \lim _{n \rightarrow \infty} f^{n}(x)=x^{*}\right\} .
$$

For a $k$-periodic point $x$, the basin of attraction is the basin of attraction $W^{s}(x)$ of $x$ under $g=f^{k}$. The basin of attraction of a $k$-cycle $c(k)=$ $\left\{x_{1}, x_{2}, \ldots, x_{k}\right\}$ is given by

$$
W^{s}(c(k))=\bigcup_{i=1}^{k} W^{s}\left(x_{i}\right)
$$

\section{Main Results}

In the sequel, $I$ denotes the compact interval $[a, b]$ and $f: I \rightarrow I$ is assumed to be continuous. Clearly if $I=[a, \infty)$ and $f$ is bounded and continuous, $f(I) \subset K \subset I$, where $K$ is a compact interval, then $f: K \rightarrow K$.

The following lemma is an immediate sequences of the Intermediate Value Theorem. 
Lemma 2.1. Let $f:[a, b] \rightarrow[a, b],(b=\infty$ allowed) be continuous and let compact $[c, d] \subset[a, b]$ be such that either

(i) $f(c)>c$ and $f(d)<d$, or

(ii) $f(c)<c$ and $f(d)>d$

Then $f$ has a fixed point in $(c, d)$.

Corollary 2.2. If in Lemma 2.1, $f(d)>d$ and $(c, d)$ is fixed point-free then $f(x)>x$ for all $x \in(c, d)$.

Theorem 2.3. The following statements are equivalent.

(i) $f$ has no points of prime (minimal) period 2 .

(ii) For all $x_{0} \in I,\left\{f^{n}\left(x_{0}\right)\right\}$ converges.

Proof. (ii) $\Rightarrow$ (i) If $\left\{\bar{x}_{1}, \bar{x}_{2}\right\}$ is a 2-cycle, then $\left\{f^{n}\left(\bar{x}_{1}\right)\right\}$ does not converge as it oscillates between $\bar{x}_{1}$ and $\bar{x}_{2}$.

(i) $\Rightarrow$ (ii) Assume that there exists $x_{0} \in I$ such that $f^{n}\left(x_{0}\right)$ does not converge. In particular $x_{0}$ is not a fixed or eventually fixed point. Then its orbit $O\left(x_{0}\right)=\left\{x_{0}, x_{1}=f\left(x_{0}\right), x_{2}=f^{2}\left(x_{0}\right), \ldots\right\}$ can be partitioned into two sequences $A=\left\{x_{k}: f\left(x_{k}\right)>x_{k}\right\}$ and $B=\left\{x_{k}: f\left(x_{k}\right)<x_{k}\right\}$. We claim that $A$ is strictly monotonically increasing, i.e. $l<j \Rightarrow x_{l}<x_{j}$. So assume that there exist $x_{l}, x_{j} \in A$ such that $l<j$ and $x_{l}>x_{j}$. This means that $f^{l}\left(x_{0}\right)>f^{j}\left(x_{0}\right)$. Let $j=l+r$. Then $f^{r}\left(x_{l}\right)<x_{l}$. Since $f^{r}(a) \geq a$, it follows from Lemma 2.1 that there exists a fixed point $z \in\left[a, x_{l}\right), f^{r}(z)=z$. Since there are no 2-cycles, by Sharkovsky's Theorem there are no cycles of periods greater than one. Hence $f(z)=z$ and $z$ is thus a fixed point. Since $x_{l}$ is not a fixed point, there exists $\delta>0$ with $\left(x_{l}-\delta, x_{l}\right)$ free of fixed points. Thus one may conclude that there exists a largest fixed point in $\left[a, x_{l}\right]$. Without loss of generality, let $z$ be such a point. From corollary 2.2, $f(x)>x$ for all $x \in\left(z, x_{l}\right)$.

Since $z$ is fixed and $f$ is continuous it follows that exists a point $y \in\left(z, x_{l}\right)$ sufficiently close to $z$ such that $f(y), f^{2}(y), \ldots, f^{r}(y) \in\left(z, x_{l}\right)$. But then we have $f^{r}(y)>y$. This together with $f^{r}\left(x_{l}\right)=x_{m}<x_{l}$ and Lemma 2.1 implies the existence of a fixed point in $\left(y, x_{l}\right)$, a contradiction which establishes the above "claim". 
Similarly, one may show that the subsequence $B$ is monotonically decreasing. Thus define

$$
x_{1}^{*}=\sup A, \quad x_{2}^{*}=\inf B .
$$

Since $A \cup B=O\left(x_{0}\right),\left\{x_{1}^{*}, x_{2}^{*}\right\}$ contains all the limit points $\Omega$ of $O\left(x_{0}\right)$. If $\left\{x_{1}^{*}, x_{2}^{*}\right\}=\Omega$ then $f\left(x_{1}^{*}\right)=x_{2}^{*}$ and $f\left(x_{2}^{*}\right)=x_{1}^{*}$, and thus $\left\{x_{1}^{*}, x_{2}^{*}\right\}$ is a 2-cycle which is ruled out by hypothesis $(i)$. The only remaining case is that $\Omega$ is a singleton which contradicts our initial assumption that the orbit of $x_{0}$ does not converge thus proving the theorem.

We remark here that a special case of this theorem was proved by Cull in [2] for one-dimensional population models. Theorem 2.3, however, applies to all continuous maps on closed intervals.

Corollary 2.4. Suppose that $f$ has a $2^{k}$-cycle but no $2^{k+1}$ cycles. Then every orbit in I converges to a periodic point (including fixed points).

Proof. Let $g=f^{2^{k}}$. Then $g$ has no 2-cycles. By Theorem 2.3, $\lim _{n \rightarrow \infty} g^{n}\left(x_{0}\right)$ converges to a fixed point $\bar{x}$ of $g$ in $I$. Thus $\bar{x}$ is $2^{r}$-periodic with $0 \leq r \leq$ $k$.

The following definition and theorem are not restricted to the real line and will be stated for general metric spaces.

Definition 2.5. Let $(X, d)$ be a metric space and $f: X \rightarrow X$ be a continuous map. A fixed point $x^{*}$ of $f$ is repelling if there exists $\varepsilon_{0}>0$ such that for all $\varepsilon, \delta, 0<\delta<\varepsilon<\varepsilon_{0}$ and $x$ such that $0<d\left(x, x^{*}\right)<\delta$, there exists $n=n\left(x_{0}, x, \delta, \varepsilon\right) \in \mathbb{Z}^{+}$such that

$$
d\left(f^{n}(x), x^{*}\right)>\varepsilon
$$

A repelling $k$-periodic point is a repelling fixed point for $f^{k}$.

Clearly, a repelling fixed point is isolated, not only as a fixed point but it is maximal in the sense that it is the largest invariant set in some neighborhood of itself, Sacker [11]. The next theorem is a direct consequence of the above definition and says that the only "approach" to a repeller is a collision.

Theorem 2.6. Let $(X, d)$ be a metric space and $f: X \rightarrow X$ be a continuous map. Let $x_{0} \in X$ be such that $\lim _{n \rightarrow \infty} f^{n k}\left(x_{0}\right)=\bar{x}$ where $\bar{x}$ is a repelling $k$ periodic point. Then $x_{0}$ is an eventually $k$-periodic point. 
Corollary 2.7. Under the assumptions of Collary 2.4, the basin of attraction of a unique asymtotically stable $2^{k}$-cycle is

$$
I \backslash E
$$

where $E$ is the set of all eventually $2^{r}$-periodic points for $0 \leq r<k$.

\section{Quasi-Unimodal Maps}

In this section we define a class of maps which is closely related to unimodal [3] [1] and which includes the logistic map and Ricker's map for certain values of their respective parameters.

Definition 3.1. A bounded continuous map $f:[0, b] \rightarrow \mathbb{R}^{+}, b \leq \infty$, is called Quasi-Unimodal (QU) if

1. $f(0)=0$, and $f(x)>0,0<x<b$

2. $\sup f$ on $[0, b]$ is attained: $f\left(x_{m}\right)=\max f$ on $[0, b]$.

3. $f$ is strictly increasing on $\left[0, x_{m}\right]$ and strictly decreasing on $\left[x_{m}, b\right]$.

4. $f(x) \rightarrow 0$ as $x \rightarrow b$.

5. $f(x)>x$ for some $x \in\left(0, x_{m}\right)$ and $f(p)=p$ for a unique point $p \in$ $(0, b)$.

The presence of multiple interior fixed points is precluded by Assumption 5. This is only assumed for convenience since only one pair of fixed points can be separated by $x_{m}$ and it is this separation property that is essential.

The following property of QU maps is useful and can be ascertained by direct inspection of the graph of the map.

Lemma 3.2. Let $f$ be a $Q U$ map. Then for all $y \in\left(0, f\left(x_{m}\right)\right), f^{-1}(y)$ consists of exactly two points, $y_{-}$and $y_{+}$, which we call conjugate points, with $y_{-}<x_{m}<y_{+}$. Further, if $0<z<y$ then

$$
z_{-}<y_{-}<x_{m}<y_{+}<z_{+}
$$

Definition 3.3. A start point [5] is a point $x$ such that $f^{-1}(x)=\emptyset$. 
Notice that if a map $f$ is quasi-unimodal, then every $x>f\left(x_{m}\right)$ is a start point.In particular, for the Ricker's model $R$, with $p>0$, every point $x>e^{p-1}$ is a start point. Moreover, for the fixed point $\mathrm{p}$, if $0<p<1$ then $p_{-1} \doteq R^{-1}(p) \backslash\{p\}$ is a start point. Our aim is to define the notion of a negative semi-orbit. Clearly, if $a \in X$ and $f$ is one-to-one then defining $a_{-n}=$ $f^{-n}(a)$ one has a unique negative semi-orbit $O^{-}(a)=\left\{\ldots, a_{-2}, a_{-1}, a\right\}$.

For many-to-one maps we have the following:

Definition 3.4. Let $a \in X$. If there exists a sequence $\left\{\ldots, a_{-2}, a_{-1}, a_{0}=a\right\}$ such that $f\left(a_{j}\right)=a_{j+1} \neq a_{j}$ then

$$
O^{-}(a)=\left\{\ldots, a_{-2}, a_{-1}, a_{0}=a\right\}
$$

is called a full negative orbit of a.

Note 1: $O^{-}(a)$ is just an element in the inverse limit [5], [8].

$$
\lim _{\longleftarrow}(X, f)
$$

of the inverse system of spaces and maps

$$
\cdots \stackrel{f}{\rightarrow} X \stackrel{f}{\rightarrow} X \stackrel{f}{\rightarrow} X
$$

after one identifies all sequences $\left\{a_{j}\right\}$ with $a_{-j}=a_{0}$, for all $j \leq M$ for some $M$.

Let $p$ be a fixed point of the map $f$. We wish to explore those points that are eventually fixed at $p$. There are two cases to consider.

Case $1 p \leq x_{m}$

Then the point $p_{+}$conjugate to $p$ satisfies $p_{+}>x_{m}$. Then from the last two properties of QU maps, $f(x)<x$ for all $x>p$ and in particular $f\left(x_{m}\right)<$ $x_{m}<p_{+}$which implies $p_{+} \in S$. Thus no full negative orbit exists. If $p=x_{m}$, then $p$ is a start point.

Case $2 p>x_{m}$

We shall define $O^{-}(p)$ by a recursive process which along the way yields a uniqueness criterion. By Lemma 3.2

$$
f^{-1}(p)=\left\{p_{-1}, p\right\}, \quad p_{-1}<x_{m}<p
$$

Deleting the "right hand" point we form

$$
f^{-1}\left(p_{-1}\right)=\left\{p_{-2}, q_{-2}\right\}, \quad p_{-2}<p_{-1}<x_{m}<p<q_{-2}
$$


Before continuing we note that at this stage we can give a condition which guarantees uniqueness of the full orbit

Condition $\mathcal{U}: \quad q_{-2}>f\left(x_{m}\right)$ This condition implies that $q_{-2} \in S$ and therefore cannot seed any further negative orbits. Continuing to ignore the right hand point

$$
f^{-1}\left(p_{-n+1}\right)=\left\{p_{-n}, q_{-n}\right\}, \quad q_{-n} \in S
$$

we thus have constructed

$$
O^{-}(p)=\left\{\ldots, p_{-2}, p_{-1}, p\right\}
$$

We now state this as a theorem.

Theorem 3.5. Let $f:[0, b] \rightarrow \mathbb{R}^{+}, b \leq \infty$ be a quasi-unimodal map with fixed point $p$ and maximum attained at $x_{m}$. Then if $p>x_{m}$, we have

1. $p$ generates a full negative orbit (3.3) and $p_{-n} \rightarrow 0$ monotonically.

2. If $q_{-2}$ defined by (3.1) and (3.2) satisfies Condition $\mathcal{U}$ then (3.3) is unique and we have further

(a) For each $n \in \mathbb{Z}^{+}$, there exists a unique monotonically increasing sequence of $n$-eventually fixed points $q_{-n}$ such that $\lim _{n \rightarrow \infty} q_{-n}=b$ and $f\left(q_{-n}\right)=p_{-n+1}$

(b) The set of eventual fixed points $E F_{p}=O^{-}(p) \cup\left\{q_{-n}: n \geq 2\right\}$

We now turn to a specific example.

\section{The Ricker map}

If $x_{n}$ represents the population size of a certain fish at time period $n$, then the difference equation

$$
x_{n+1}=R\left(x_{n}\right),
$$

where $R(x)=x e^{p-x}$ on $[0, \infty)[10]$, is the model used by Ricker to study a variety of fish and invertebrate populations including Pacific herring, the pink salmon, the haddock, the fruit fly, the water flea, and the starfish among others. The Ricker's map is bounded and attains its maximum value $b=e^{p-1}$. 
Thus we may consider $R:[0, b] \rightarrow[0, b]$. Hence Corollary 2.7 applies to the Ricker's model. We have thus proved Elaydi-Yakubu Conjecture [6]. However, we are going to prove the conjecture using more elementary techniques and at the same time delve more deeply into the fine structure of the basin of attraction. There are two fixed points: $x_{1}^{*}=0, x_{2}^{*}=p$. We now discuss the stability of these fixed points [4].

1. $R^{\prime}(x)=(1-x) e^{p-x}, R^{\prime \prime}(x)=(x-2) e^{p-x}, R^{\prime \prime \prime}(x)=(3-x) e^{p-x}$

2. $R^{\prime}(0)=e^{p}>1$ and thus 0 is unstable

3. $R^{\prime}(p)=(1-p)$ and thus $p$ is stable if $0<p<2$. For $p=2, R^{\prime}(p)=-1$ and we need further analysis to determine the stability of $\mathrm{p}$.

The Schwarzian derivation of $R$ is given by

$$
S R=\frac{R^{\prime \prime \prime}(p)}{R^{\prime}(p)}-\frac{3}{2}\left[\frac{R^{\prime \prime}(p)}{R^{\prime}(p)}\right]^{2}=\frac{-x^{2}+4 x-6}{2(1-x)^{2}}<0
$$

for all $x$. Hence $x_{2}^{*}=2$ is asymptotically stable [4]. When $p>2, x_{2}^{*}$ loses its stability and an asymptotically stable 2-cycle $\left\{\bar{x}_{1}, \bar{x}_{2}\right\}$ is born which in turn loses its stability past $p_{2} \approx 2.5264$. It is important to note that the new born $2^{k}$-cycles are unique.

\subsection{Eventually fixed points}

If $0<p<1$, then $R^{-1}(p)=\left\{p, q_{-1}\right\}$, where $q_{-1}$ is a start point. And if $p=1$, then $R^{-1}(p)=p$. Finally, let us now consider the case where $1<p \leq 2$. It is easily checked that Ricker's map is quasi-unimodal.

It only remains to verify that Condition $\mathcal{U}$ is satisfied.

Lemma 4.1. For $1<p \leq 2$, Ricker's map satisfies Condition $\mathcal{U}$.

Proof. For $R(x)=x e^{p-x}$ we must verify $q_{-2}>R(1)$. Assume not, i.e. $R(1) \geq q_{-2}$. Since the maximum of $R$ is attained at $x_{m}=1$, it follows from Lemma 3.1 and 3.2 that $q_{-2}>1$. Also $p>1$ gives us $e^{p-1}>1$. Thus since $R$ is decreasing in $[1, \infty)$, applying $R$ to the assumed inequality,

$$
R^{2}(1) \leq R\left(q_{-2}\right)=p_{-1}<1
$$


and therefore $R\left(e^{p-1}\right)<1$. But

$$
R\left(e^{p-1}\right)=e^{p-1} e^{p-e^{p-1}}=e^{2 p-1-e^{p-1}}
$$

Defining $h(t)=2 t-1-e^{t-1}$, we will show $h(t)>0$ on $(0,2]$ to obtain a contradiction. Note $h(1)=0$ and $h^{\prime}(t)=2-e^{t-1}$ so that $h^{\prime}(1)=1$. The maximum of $h$ is attained at $t_{0}=1+\ln 2<2$ and $h^{\prime}(t)<0$ in $\left(t_{0}, 2\right]$. Therefore the minimum of $h$ in that interval is at 2 . But $h(2)=4-1-e>0$ which proves the lemma. Thus all the conclusions of Theorem 3.5 follow.

\subsection{Eventually 2-periodic points}

We now assume that $2<p<p_{2}$, where $p_{2} \approx 2.5264$, so we have an attracting 2-cycle $\{\bar{x}, \bar{y}\}$ with $\bar{x}<p<\bar{y}$.

Theorem 4.2. The basin of attraction of the 2-cycle $\{\bar{x}, \bar{y}\}, 2<p<p_{2}$, where $p_{2} \approx 2.5264$, is

$$
W^{s}(\{\bar{x}, \bar{y}\})=(0, \infty) \backslash E F
$$

where EF is the set of all eventually fixed points of $R$.

The proof consists of several lemmas.

Lemma 4.3. Let $f: X \rightarrow X$ be a continuous map on a metric space $X$. Then for an asymptotically stable fixed point $x^{*}$ of $f$, the component $C$ in $W^{s}\left(x^{*}\right)$ containing $x^{*}$ and its boundary $\partial C$ are each invariant.

From Theorem 3.5 we have a full negative orbit $O^{-}(p)=\left\{\ldots, p_{-2}, p_{-1}, p\right\}$ with $p_{-n} \rightarrow 0$ monotonically. We shall use this to prove the following

Lemma 4.4. Let $\left(a_{1}, b_{1}\right)$ be the component of $W^{s}(\bar{x})$ containing $\bar{x}$ and $\left(c_{1}, d_{1}\right)$ be the component of $W^{s}(\bar{y})$ containing $\bar{y}$. Then

(i) $b_{1}=c_{1}=p$

(ii) $a_{1}=p_{-1}, d_{1}=q_{-2}$

Proof. By Lemma 4.3, the sets $\left\{a_{1}, b_{1}\right\}$ and $\left\{c_{1}, d_{1}\right\}$ are invariant under $R^{2}$. If $R^{2}\left(a_{1}\right)=a_{1}$, then either $a_{1}$ is a 2-periodic point, which violates uniqueness of the 2-periodic orbit, or $a_{1}$ is a fixed point, namely $a_{1}=0$. But by this is impossible since the eventually fixed points $p_{-n} \rightarrow 0$ and therefore $p_{-n} \in$ 
$\left(a_{1}, b_{1}\right)$ for some large $n$. Thus $R^{2}\left(a_{1}\right)=b_{1}$. If $R^{2}\left(b_{1}\right)=a_{1}, a_{1}$ is a 4-cycle which is absurd since a 4 -cycle (which is not a 2-cycle) consists of 4 distinct points. Hence $f^{2}\left(b_{1}\right)=b_{1}$. Since $b_{1} \leq p$ and $b_{1}$ cannot be a 2 -periodic point, $b_{1}=p$.

The argument that $c_{1}=p$ is similar: Using uniqueness and invariance

$$
R^{2}\left(d_{1}\right)=d_{1} \Rightarrow R\left(d_{1}\right)=d_{1} \Rightarrow d_{1} \text { fixed }
$$

a contradiction. Thus $R^{2}\left(d_{1}\right)=c_{1}$. If $R^{2}\left(c_{1}\right)=d_{1}$, then $R^{4}\left(d_{1}\right)=d_{1} \Rightarrow$ $R^{2}\left(d_{1}\right)=d_{1}$ which again leads to a contradiction. Thus we must have $R^{2}\left(c_{1}\right)=c_{1}$ which implies $c_{1}=p$, i.e. $R^{2}\left(d_{1}\right)=p$.

Finally since by Lemma 3.2, $R^{-1}(p)=\left\{p_{-1}, p\right\}$ with $p_{-1}<1$ and therefore $R\left(d_{1}\right) \neq p$ which implies that $R\left(d_{1}\right)=a_{1}$ (Note, The set $\left\{a_{1}, b_{1}, c_{1}, d_{1}\right\}$ is invariant under $R$ ). Hence $p=R^{2}\left(d_{1}\right)=R\left(a_{1}\right)$. Thus $a_{1}=p_{-1}$ and $d_{1}=q_{-2}$.

Lemma 4.5. (i) The full negative orbit $O^{-}(p)$ is unique.

(ii) The sequence $\left\{q_{-n}\right\}$ monotonically diverges to $\infty$ and each $q_{-n}$ is a start point.

Proof. By virtue of Theorem 2.3, it suffices to prove that $q_{-2}>R(1)$. So assume that $q_{-2} \leq R(1)$. Then since $q_{-2}>1$ and $R$ is decreasing there,

$$
p_{-1}=R\left(q_{-2}\right) \geq R^{2}(1)
$$

But by Lemma 4.4 , since $1 \in\left(p_{-1}, p\right), R^{2}(1) \in\left(p_{-1}, p\right)$ which contradicts (4.1).

Consider now the map $G=R^{2}=R \circ R$. Then $\mathrm{G}$ has three critical points $x_{1}<x_{2}<x_{3}$, where $x_{2}=1$, and $x_{1}, x_{3} \in R^{-1}(1)$, that is $x_{i} e^{p-x_{i}}=1$, for $i=1,3$. There is a parameter value $p_{c} \approx 2.25643$ at which $\bar{x}=1$ and $\bar{y}=e^{p_{c}-1}$. In addition to the case $p=p_{c}$, there are two cases to consider.

1. If $2<p<p_{c}$, then we have $\bar{x}>1$ and $\bar{y}<x_{3}$.

2. If $p>p_{c}$, then we have $\bar{x}<1$ and $\bar{y}>x_{3}$.

The next lemma investigates these three cases.

Lemma 4.6. The following statements hold true for the 2-cycle $\{\bar{x}, \bar{y}\}$. 
(i) If $2<p<p_{c}$, then $G^{-1}(\bar{x})=\left\{x_{-1}^{1}, x_{-1}^{2}, x_{-1}, \bar{x}\right\}$, where $x_{-1} \in\left(q_{-2}, q_{-3}\right)$ is a start point, and $x_{-1}^{1} \in\left(p_{-1}, \bar{x}\right), x_{-1}^{2} \in\left(p_{-3}, p_{-2}\right)$. Moreover, $G^{-1}(\bar{y})=\left\{y_{-1}^{1}, y_{-1}^{2}, y_{-1}, \bar{y}\right\}$, with $y_{-1} \in\left(\bar{y}, q_{-2}\right)$ is a start point, $y_{-1}^{1}, y_{-1}^{2} \in$ $\left(p_{-2}, p_{-1}\right)$.

(ii) If $p=p_{c}$, then $G^{-1}(\bar{x})=\left\{x_{-1}^{1}, x_{-1}, \bar{x}\right\}$, where $x_{-1} \in\left(q_{-2}, q_{-3}\right)$ is a start point, and $x_{-1}^{1} \in\left(p_{-3}, p_{-2}\right)$. Moreover, $G^{-1}(\bar{y})=\left\{y_{-1}, \bar{y}\right\}$, with $y_{-1} \in\left(p_{-2}, p_{-1}\right)$.

(iii) If $p>p_{c}$, then $G^{-1}(\bar{x})=\left\{x_{-1}^{1}, x_{-1}^{2}, x_{-1}, \bar{x}\right\}$, where $x_{-1} \in\left(q_{-2}, q_{-3}\right)$ is a start point, and $x_{-1}^{1} \in(\bar{x}, p), x_{-1}^{2} \in\left(p_{-3}, p_{-2}\right)$. Moreover, $G^{-1}(\bar{y})=$ $\left\{y_{-1}^{1}, y_{-1}^{2}, y_{-1}, \bar{y}\right\}$, with $y_{-1} \in(p, \bar{y})$, and $y_{-1}^{1}, y_{-1}^{2} \in\left(p_{-2}, p_{-1}\right)$.

Proof. (i) Claim that $q_{-2}>x_{3}$. For if $q_{-2} \leq x_{3}$, then $p_{-1}=R\left(q_{-2}\right) \geq$ $R\left(x_{3}\right)=1$ which contaradicts the results in Lemma 3.2. Since $R^{2}$ is decreasing on the interval $\left(x_{3}, \infty\right)$, there exists $x_{-1}>q_{-2}$ with $R^{2}\left(x_{-1}\right)=\bar{x}$. Since $R^{3}\left(x_{-1}\right)=\bar{y}$ and $R^{3}\left(q_{-3}\right)=p$, it follows that $x_{-1}<q_{-3}$. The proof of the remaining parts is straightforward and will be omitted.

The scenario in Lemma 4.6 may be extended to higher order cycles.

Proof of Theorem 4.2: Let $\left(p_{-n}, p_{-n+1}\right)$ be an interval as defined in section 2. Since $p_{-n}<1$ for all $n=1,2,3, \ldots$ the map $R$ is strictly increasing on $\left[p_{-n}, p_{-n+1}\right]$. It follows that $R\left(\left[p_{-n}, p_{-n+1}\right]\right)=\left[p_{-n+1}, p_{-n+2}\right]$. Thus $R^{n-1}\left(\left[p_{-n}, p_{-n+1}\right]\right)=\left[p_{-1}, p_{0}\right]$. This implies by Lemma L-2-bas that $R^{n-1}\left(\left(p_{-n}, p_{-n+1}\right)\right)=\left(p_{-1}, p_{0}\right)=W^{s}(\bar{x})$. Hence $\left(p_{-n}, p_{-n+1}\right) \subset W^{s}(\{\bar{x}, \bar{y}\})$ since $q_{-n}>1$, for $n \geq 2, R$ is strictly decreasing on $\left[q_{-n}, q_{-n-1}\right]$. Hence $R^{n-1}\left(\left[q_{-n}, q_{-n-1}\right]\right)=\left[p_{-2}, p_{-1}\right]$ and consequently, $R^{n}\left(q_{-n}, q_{-n-1}\right)=\left(p_{-1}, p\right)=$ $W^{s}(\bar{x})$. Hence $\left(q_{-n}, q_{-n-1}\right) \subset W^{s}(\{\bar{x}, \bar{y}\})$.

Theorem 4.7. Let $c_{k}=\left\{\bar{x}_{1}, \bar{x}_{2}, \ldots, \bar{x}_{2^{k}}\right\}$ be asymptotically stable $2^{k}$-cycle. Then $W^{s}\left(c_{k}\right)$ is $(0, \infty) \backslash \bigcup_{i \in \Lambda} E P_{\bar{x}_{i}}$, where $\left\{\bar{x}_{i}: i \in \Lambda\right\}$ is the set of all eventually $2^{r}$-periodic points $0 \leq r<k$.

\section{References}

[1] L.S. Block and W.A. Coppel, Dynamics in one dimension, Lecture Notes in Mathematics, Vol. 1513, Springer-Verlag, Berlin, 1992. 
[2] P. Cull , Global stability of population models, Bull. math. Biol. 43 (1981), 47-58.

[3] Robert L. Devaney, An introduction to chaotic dynamical systems, Benjamin/Cummings Publ., 1986.

[4] S. Elaydi, Discrete Chaos, Chapman \& Hall/CRC, Boca Raton, 2000.

[5] S. Elaydi , Semidynamical systems with nonunique global backward extensions, Funkcial. Ekvac. 26(2) (1983), 115-230.

[6] S. Elaydi and A. Yakubu, Basins of attraction of stable cycles, J. Diff. Equations Appl. 8(8) (2002), 755-760.

[7] S. Elaydi and A. Yakubu, Global stability of cycles: Lotka-Volterra competition model with stocking, J. Diff. Equations Appl. 8(6) (2002), 537549.

[8] John G. Hocking and Gail S. Young, Topology, Addison-Wesley, 1961; Dover, 1988.

[9] R.M. May, Biological populations with nonoverlapping generations: stable points, stable cycles, and chaos, Science 186 (1974), 645-647.

[10] W.E. Ricker, Stock and Recruitment, J. Fish. Res. Bd. Canada 11(5) (1954), 559-620.

[11] Robert J. Sacker, Asymptotic approach to periodic orbits and local prolongations of maps, Pacific J. Math. 51(1) (1974), 273-287.

Department of Mathematics, Trinity University, San Antonio, TX 78212, selaydi@trinity.edu.

Department of Mathematics, University of Southern California, Los Angeles, CA 90089-1113, rsacker@math.usc.edu-http://math.usc.edu/ rsacker 